\title{
Clinical Features of Geriatric Syndromes in Older Koreans with Diabetes Mellitus
}

\author{
Shinje Moon ${ }^{1}$, Yong Kyun Roh ${ }^{2}$, Jong Lull Yoon ${ }^{3}$, Ki Un Jang ${ }^{4}$, Hun Jong Jung ${ }^{5}$, Hyung Joon Yoo ${ }^{6}$, Hak Chul Jang ; \\ Geriatric Syndromes Study Group of Korea Geriatrics Society \\ ${ }^{1}$ Division of Endocrinology and Metabolism, Department of Internal Medicine, Kangnam Sacred Heart Hospital, Hallym University College of Medicine, Seoul, \\ Korea \\ ${ }^{2}$ Department of Family Medicine, Kangnam Sacred Heart Hospital, Hallym University College of Medicine, Seoul, Korea \\ ${ }^{3}$ Department of Family Medicine, Dongtan Sacred Heart Hospital, Hallym University College of Medicine, Hwaseong, Korea \\ ${ }^{4}$ Department of Rehabilitation Medicine, Kangnam Sacred Heart Hospital, Hallym University College of Medicine, Seoul, Korea \\ ${ }^{5}$ Department of Occupational and Environmental Medicine, Konkuk University Chungju Hospital, Chungju, Korea \\ ${ }^{6}$ Division of Internal Medicine, CM Hospital, Seoul, Korea \\ ${ }^{7}$ Department of Internal Medicine, Seoul National University Bundang Hospital, Seongnam, Korea
}

\section{Corresponding Author:}

Hak Chul Jang, MD, PhD

Department of Internal Medicine,

Seoul National University Bundang

Hospital, Seoul National University

College of Medicine, 82, Gumi-ro

173beon-gil, Bundang-gu, Seongnam

13620, Korea

E-mail: janghak@snu.ac.kr

ORCID:

https://orcid.org/0000-0002-4188-6536

Received: November 23, 2019

Revised: December 13, 2019

Accepted: December 16, 2019
Background: This study explored the prevalence and clinical characteristics of geriatric syndromes among Korean older adults with diabetes mellitus (DM). Methods: We used data from the 2017 National Survey of Older Koreans to analyze the classic geriatric syndromes of polypharmacy, urinary incontinence, falls, cognitive impairment, and functional impairment according to the presence of DM. Results: Among 10,299 participants aged 65 years or older, 2,395 had DM. The prevalence of polypharmacy was $64.1 \%$ in the DM group and $31.6 \%$ in the non-DM group ( $p<0.001)$. One or more falls per year occurred in $18.7 \%$ of participants with DM compared with $14.9 \%$ of those without $D M(p<0.001)$. The prevalence of urinary incontinence was significantly higher in the DM group (3.8\%) than in the non-DM group (2.5\%) $(\mathrm{p}=0.001)$. The prevalence of cognitive impairment was $17.7 \%$ in the DM group versus $14.9 \%$ in the non-DM group $(p=0.001)$. Functional impairment occurred in $32.2 \%$ of participants in the DM group compared with $26.8 \%$ of participants in the non-DM group $(p<0.001)$. Finally, the number of geriatric syndromes was significantly associated with cardiovascular disease (CVD) and chronic kidney disease (CKD) in patients with DM. Conclusion: The results of this study showed a higher prevalence of geriatric syndromes among older Korean adults with DM. In addition, the coexistence of multiple geriatric syndromes was associated with CVD and CKD among patients with DM. These findings support the current guidelines for older adults with DM that recommend assessment for geriatric syndromes.

Key Words: Diabetes mellitus, Geriatric syndrome, Geriatric assessment

\section{INTRODUCTION}

The life expectancy is increasing worldwide, and Korea has one of the fastest aging populations. ${ }^{1-3)}$ According to national statistics, the proportions of the population aged 65 years or older were $3.8 \%$ in $1990,7.2 \%$ in 2000 , and $14.2 \%$ in $2017^{2,3)}$ This proportion is projected to reach $25 \%$ in 2030 , marking the beginning of a super-aged society, ${ }^{2,3)}$ With the rapid increase in the number of older adults, the incidence of diabetes mellitus (DM) is also increasing steeply. According to the International Diabetes Federation, 10 million patients were newly diagnosed with diabetes between 2015 and 2017, 8 million of whom were aged 65 years or older. ${ }^{4)}$ 
In Korea, the prevalence of DM in people aged $\geq 65$ years was $30 \%$ in $2016^{3,5,6)}$

The treatment of DM in older adults is often difficult because of their impaired physical, psychological, and cognitive functions. ${ }^{7)}$ Moreover, older adults with DM have an increased risk of premature death and comorbid diseases such as hypertension, coronary heart disease, and stroke compared with individuals without diabetes. ${ }^{8,9)}$ Also, atypical symptoms occur in older adults with DM because of multi-morbidity and polypharmacy. ${ }^{6-8,10,11)}$ Therefore, a comprehensive approach to various symptoms is necessary for the treatment of older adults with DM, for which comprehensive assessment of geriatric syndromes is needed. ${ }^{11,12)}$ Geriatric syndromes refer to multi-factorial conditions among older adults that render them vulnerable to situational changes. ${ }^{13)}$ Geriatric syndromes are highly prevalent and are associated with a high morbidity and poor quality of life. ${ }^{13)}$

Considering the variability in symptoms among older adults with DM due to multiple comorbidities and disabilities, the American Diabetes Association recommends screening for geriatric syndromes such as polypharmacy, cognitive impairment, urinary incontinence, falls, and persistent pain in addition to diabetes self-management and health-related quality of life among older adults with DM. ${ }^{8)}$ The Korean Diabetes Association also recommends individual assessment of geriatric syndromes such as polypharmacy, cognitive impairment, and functional impairment among older adults with DM and that the outcomes of such assessments be reflected in the diabetes treatment plan. ${ }^{5)}$

Therefore, the assessment of geriatric syndromes in older adults with DM is important. However, little is known regarding the demographic and clinical characteristics of geriatric syndromes among older adults with DM in Korea. Thus, the present study explores the prevalence and clinical characteristics of geriatric syndromes among older adults with DM on the basis of data from the 2017 National Survey of Older Koreans (NSOK). ${ }^{14)}$

\section{MATERIALS AND METHODS}

\section{Study Population}

The NSOK is a cross-sectional, nationwide mandatory survey performed every 3 years since $2008 .{ }^{14)}$ The purpose of the NSOK is to provide baseline information to formulate policies related to older adults on the basis of a legal provision (Welfare of Older Persons Act). The NSOK investigates the socioeconomic status, health condition, and needs and desires of older Koreans via in-person interviews conducted by specialized surveyors. This study used data from the fourth NSOK survey, conducted in 2017 by the Korea Institute for Health and Social Affairs, which included 10,299 old- er adults aged $\geq 65$ years in 934 survey areas from June 12 to August 28, 2017.

\section{Assessment of Geriatric Syndromes}

This study analyzed the classic geriatric syndromes of polypharmacy, urinary incontinence, falls, cognitive impairment, and functional impairment. ${ }^{7,8)}$ Polypharmacy was defined as the prescription of 5 or more drugs for 3 months or longer. ${ }^{15)}$ Urinary incontinence was defined as the presence of diagnosed incontinence for 3 months or longer. Falls were defined as the occurrence of one or more falls over 1 year, whereas recurrent falls were defined as the occurrence of two or more falls per year. Cognitive function was assessed using the Korean version of the Mini-Mental Status Examination for dementia screening (MMSE-DS), with cognitive impairment determined on the basis of a cutoff score adjusted for sex, age, and educational level. Physician-diagnosed Alzheimer disease was also categorized as cognitive impairment. Functional status in the older adults was assessed on the basis of 7 categories of activities of daily living (ADLs) and 10 categories of instrumental activities of daily living (IADLs), ${ }^{14,16,17)}$ with functional impairment defined as the presence of one or more difficulties in $\mathrm{ADL}$ and IADL. ${ }^{14)}$ Visual and hearing status was assessed via a structured questionnaire, with impairment defined as any inconvenience in daily life such as watching TV, reading the newspaper, talking on the phone, and talking with a person or the need for assistance due to visual and hearing impairment. Participants with limitations in sitting and rising from a chair or bed 5 times with both hands in front (without using both hands to assist in the task) were considered to have lower limb limitations. Depression was assessed using the Korean version of the Geriatric Depression ScaleShort Form which comprised 15 questions, with depression defined as a score $\geq 8$ or a prior diagnosis of depression. ${ }^{14)}$

\section{Definition of Diabetes and Diabetic Complications}

DM was defined as the presence of diagnosed diabetes for 3 months or longer. Cardiovascular diseases (CVDs), including angina, myocardial infarction, and stroke and chronic kidney disease (CKD), were analyzed as diabetic complications. The presence of a definite diagnosis was assessed using a structured questionnaire.

\section{Statistical Analysis}

The data are presented as means with standard deviations or as frequencies with percentages according to the distribution of DM. Statistical analyses were performed using the t-test and Pearson chi-squared test. The odds ratios (ORs) of geriatric syndromes for diabetic complications were assessed via multivariate logistic regression analysis. Statistical analyses were performed using IBM 
SPSS Statistics for Windows version 24.0 (IBM Corp., Armonk, NY, USA). Analysis items with $\mathrm{p}<0.05$ were considered statistically significant.

\section{Ethical Considerations}

The study was conducted in accordance with the tenets of the Declaration of Helsinki. All NSOK protocols were approved by the Institutional Review Board of the Korea Institute for Health and Social Affairs (No. 2017-11). All participants volunteered and provided written informed consent before their enrollment. All participants' records were anonymized before accession by the authors and all procedures were performed according to approved guidelines and regulations.

\section{RESULTS}

\section{Baseline Characteristics}

This study included 10,299 participants (4,120 men and 6,179 women) aged 65-106 years, 2,395 (928 men and 1,467 women) of whom had DM. Table 1 shows the baseline characteristics of participants in this study according to diabetic status. No significant differences in age, sex, and smoking status were observed. However, participants with DM had a higher body mass index and prevalence of hypertension, dyslipidemia, CKD, and CVD than did participants without DM. The DM group had more comorbidities than did the non-DM group $(\mathrm{p}<0.001)$.

\section{Geriatric Syndromes among Older Adults according to DM Status}

Patients with DM were prescribed an average of 6.2 medications, which was significantly higher than that in the non-DM group (3.4 medications) $(\mathrm{p}<0.001)$ (Table 2$)$. The prevalence of polypharmacy ( $\geq 5$ prescribed medications during $\geq 3$ months) was $64.1 \%$ in the DM group and $31.6 \%$ in the non-DM group $(\mathrm{p}<0.001)$. One or more falls per year observed in $18.7 \%$ of participants in the DM group compared with $14.9 \%$ of participants in the non-DM group and the incidence of recurrent falls ( $\geq 2$ falls per year) was also significantly higher in the DM group $(\mathrm{p}<0.001)$. The prevalence of urinary incontinence was significantly higher in the DM group $(3.8 \%)$ than in the non-DM group $(2.5 \%)(p=0.001)$. The prevalence of cognitive impairment was $17.7 \%$ in the DM group and $14.9 \%$ in the non-DM group $(p=0.001)$. Visual and hearing impairment observed in $46.6 \%$ of participants in the DM group compared with $42.7 \%$ of participants in the non-DM group $(p=0.001)$. Lower limb limitations were observed in $27.2 \%$ of participants in the DM group compared with $21.2 \%$ of participants in the non-DM group $(\mathrm{p}<0.001)$. Functional impairment, as assessed by ADL and IADL, existed in $32.2 \%$ of participants in the DM group compared with $26.8 \%$ of participants in the non-DM group $(\mathrm{p}<0.001)$. Depression existed in $14.8 \%$ of participants in the DM group compared with $10.8 \%$ of participants in the nonDM group $(\mathrm{p}<0.001)$.

\section{Association between Diabetic Complications and Geriatric Syndromes in Older Adults with DM}

A significant association was observed between CVD and polypharmacy $(\mathrm{OR}=7.01$; 95\% confidence interval [CI], 5.03-10.03) (Table 3). Participants with CVD had significantly higher ORs for falls $(\mathrm{OR}=1.44 ; 95 \% \mathrm{CI}, 1.11-1.87)$, cognitive impairment $(\mathrm{OR}=1.34 ; 95 \% \mathrm{CI}, 1.03-1.75)$, and functional impairment

Table 1. Clinical characteristics of participants from the 2017 National Survey of Older Koreans

\begin{tabular}{lccc}
\hline Characteristic & Without DM $(\mathrm{n}=7,904)$ & With DM $(\mathrm{n}=2,395)$ & $\mathrm{p}$-value \\
\hline Age $(\mathrm{y})$ & $74.6 \pm 6.5$ & $74.6 \pm 5.9$ & 0.872 \\
Sex, male & $3,192(40.5)$ & $928(39.0)$ & 0.198 \\
Years of education & $6.8 \pm 4.6$ & $6.5 \pm 4.6$ & 0.008 \\
Current smoking & $754(9.5)$ & $204(8.5)$ & 0.142 \\
Alcohol consumption & $2,029(25.7)$ & $497(20.8)$ & $<0.001$ \\
Body mass index $\left(\mathrm{kg} / \mathrm{m}^{2}\right)$ & $23.3 \pm 3.0$ & $24.1 \pm 3.1$ & $<0.001$ \\
Physical activity, $\geq 150 \mathrm{~min} / \mathrm{wk}$ & $3,627(45.9)$ & $1,085(45.3)$ & 0.631 \\
Hypertension & $4,322(54.7)$ & $1,804(75.3)$ & $<0.001$ \\
Dyslipidemia & $1,937(24.5)$ & $944(39.4)$ & $<0.001$ \\
Cancer & $276(3.5)$ & $95(4.0)$ & 0.303 \\
Cardiovascular disease & $1,041(13.2)$ & $428(17.9)$ & $<0.001$ \\
Chronic kidney disease & $90(1.1)$ & $101(4.2)$ & $<0.001$ \\
Comorbidity & $2.5 \pm 1.7$ & $4.0 \pm 1.7$ & $<0.001$ \\
\hline
\end{tabular}

Values are presented as mean \pm standard deviation or number (\%).

$\mathrm{DM}$, diabetes mellitus. 
Table 2. Characteristics of patients with geriatric syndromes according to DM status

\begin{tabular}{lccr}
\hline Characteristic & Without DM $(\mathrm{n}=7,904)$ & With DM $(\mathrm{n}=2,395)$ & $\mathrm{p}$-value \\
\hline Number of medications & $3.5 \pm 3.9$ & $6.2 \pm 4.3$ & $<0.001$ \\
Polypharmacy, $\geq 5$ medications & $2,496(31.6)$ & $1,534(64.1)$ & $<0.001$ \\
Urinary incontinence & $201(2.5)$ & $91(3.8)$ & 0.001 \\
Falls, $\geq 1$ per year & $1,173(14.9)$ & $446(18.7)$ & $<0.001$ \\
Recurrent falls, $\geq 2$ per year & $397(5.1)$ & $166(7.0)$ & $<0.001$ \\
MMSE-DS score & $25.1 \pm 3.9$ & $24.7 \pm 3.9$ & $<0.001$ \\
$\quad$ Cognitive impairment & $1,155(14.9)$ & $415(17.7)$ & 0.001 \\
Functional impairment & $2,120(26.8)$ & $771(32.2)$ & $<0.001$ \\
SGDS score & $5.1 \pm 1.9$ & $5.3 \pm 2.0$ & $<0.001$ \\
$\quad$ Depression & $838(10.8)$ & $347(14.8)$ & $<0.001$ \\
Number of coexisting geriatric syndromes* & & & $<0.001$ \\
$\quad 1$ & $2,496(32.2)$ & $921(39.3)$ & $593(25.3)$ \\
2 & $1,245(16.1)$ & $325(13.9)$ & \\
$\quad 604(7.8)$ & & \\
\end{tabular}

Values are presented as mean \pm standard deviation or number (\%).

DM, diabetes mellitus.

*Geriatric syndromes included polypharmacy, urinary incontinence, falls, cognitive impairment, and functional impairment.

$(\mathrm{OR}=1.78 ; 95 \% \mathrm{CI}, 1.40-2.27)$ (Table 3). However, CVD was not associated with urinary incontinence. Furthermore, participants with CKD had significantly higher ORs for polypharmacy $(\mathrm{OR}=3.85 ; 95 \% \mathrm{CI}, 2.11-7.00)$, urinary incontinence $(\mathrm{OR}=5.07 ; 95 \% \mathrm{CI}, 2.49-10.33)$, and functional impairment $(\mathrm{OR}=2.47 ; 95 \% \mathrm{CI}, 1.60-3.82)$ (Table 3). Finally, the number of geriatric syndromes was significantly associated with CVD and CKD in patients with DM (Table 4).

\section{DISCUSSION}

This study evaluated the prevalence of geriatric syndromes among older Korean adults with DM. Patients with DM had a significantly higher prevalence of geriatric syndromes than did participants without DM. In addition, diabetic complications such as CVD and $\mathrm{CKD}$ were associated with geriatric syndromes.

Despite its importance in clinical practice, a universal definition of polypharmacy has yet to be established. Many studies, including the present study, defined polypharmacy as the daily use of 5 or more medicines. ${ }^{15,18)}$ Although the prevalence of polypharmacy among older adults with DM varied from $6.25 \%$ to $93.4 \%, \mathrm{DM}$ is a considerable cause of polypharmacy due to the requirement of glycemic control and treatment of diabetic complications. ${ }^{18)}$ Growing evidence suggests that polypharmacy in patients with DM patients increases the number of adverse drug events such as severe hypoglycemia, drug-drug interactions, and interactions with coexisting comorbidities. ${ }^{18-24)}$ Particularly, older adults with DM are at a high risk for polypharmacy because of multi-morbidity, age-related
Table 3. Association of geriatric syndromes with cardiovascular disease and chronic kidney disease

\begin{tabular}{lcc}
\hline & Cardiovascular disease & Chronic kidney disease \\
\hline Polypharmacy & $7.10(5.03-10.03)$ & $3.85(2.11-7.00)$ \\
Falls & $1.44(1.11-1.87)$ & $1.33(0.81-2.18)$ \\
Urinary incontinence & $1.25(0.70-2.23)$ & $5.07(2.49-10.33)$ \\
Cognitive impairment & $1.34(1.03-1.76)$ & $0.93(0.54-1.60)$ \\
Functional impairment & $1.78(1.40-2.26)$ & $2.47(1.60-3.82)$
\end{tabular}

Values are presented as odds ratio (95\% confidence interval). The data were adjusted for age, sex, smoking status, alcohol consumption, body mass index, hypertension, and dyslipidemia.

Table 4. Association of the numbers of coexisting geriatric syndromes with cardiovascular disease and chronic kidney disease

\begin{tabular}{lcc}
\hline & Cardiovascular disease & Chronic kidney disease \\
\hline $\begin{array}{l}\text { Numbers of geriatric } \\
\text { syndromes }\end{array}$ & & \\
0 & Reference & Reference \\
1 & $6.05(3.70-9.90)$ & $5.02(1.77-14.25)$ \\
2 & $8.29(4.98-13.81)$ & $7.72(2.67-22.35)$ \\
$\geq 3$ & $12.28(7.18-21.00)$ & $11.272(3.80-33.40)$ \\
\hline
\end{tabular}

Values are presented as odds ratio (95\% confidence interval). The data were adjusted for age, sex, smoking status, alcohol consumption, body mass index, hypertension, and dyslipidemia.

pharmacokinetic changes in liver or kidney diseases, and non-adherence to treatment regimens. ${ }^{18,25,26)}$ The results of the present study also show a significantly higher rate of polypharmacy among older patients with DM than among non-DM participants. Notably, polypharmacy in patients with DM was associated with high 
prevalence of CVD and CKD. This result supports the guidelines of the American Diabetes Association and Korean Diabetes Association that recommend screening for polypharmacy in older patients with DM. Although the harms of polypharmacy are uniformly reported, the balance of benefits and harms has not been evaluated in randomized controlled trials. ${ }^{26)}$ Therefore, a reduction in the number of medications should be determined on the basis of individual benefits and harms.

Falls are a major geriatric syndrome that can lead to fractures, aggravation of glycemic control, and reduction in the quality of life of older persons with DM. Several studies have shown that DM is a major risk factor for falls. ${ }^{27,28)}$ The Study of Osteoporotic Fractures, which included 9,247 older women, demonstrated that women with DM had an increased risk of falling compared with non-DM women. ${ }^{27)}$ The Women's Health and Aging Study also showed that DM was associated with an increased risk of falls. ${ }^{28)}$ The present study also observed a significantly higher incidence of falls among patients with DM. The increased risk of falls may be partially explained by gait impairment in patients with $\mathrm{DM}^{29-31)}$ Moreover, our study showed higher prevalence of visual and hearing impairment, lower limb limitations, and functional impairment in older adults with DM, which might contribute to gait impairment and an increased incidence of falls.

Previous studies have reported an association between diabetes and urinary incontinence. ${ }^{32-34)}$ In a prospective study of 81,854 women, the prevalence of urinary incontinence was $24.4 \%$ among women with DM and 17.1\% among those without DM. A 1.21fold increased risk for any urinary incontinence (95\% CI, 1.021.43 ) and 1.40-fold increased risk of severe incontinence in patients with DM were also reported (95\% CI, 1.15-1.71). ${ }^{33)}$ The present study also showed the association between urinary incontinence and DM. While the prevalence of urinary incontinence identified in this study was lower than that reported previously, ${ }^{32-34)}$ it was similar to those reported in previous Korean studies. ${ }^{35,36)}$ Urinary incontinence was also significantly correlated with CKD in the present. However, because of the cross-sectional nature, a prospective study is needed to assess the causal relationship between urinary incontinence and CKD.

Various studies have reported an association between diabetes and cognitive impairment. ${ }^{37,38)}$ Epidemiological studies have observed a 1.6- to 3.0-fold increased risk of dementia among patients with DM. ${ }^{39,40)}$ Hyperglycemia, advanced glycation end products, and hyperinsulinemia or insulin resistance are associated with the pathophysiological mechanisms underlying cognitive impairment. ${ }^{37,38,41-43)}$ We also observed a higher prevalence of cognitive impairment among patients with DM. Moreover, cognitive impairment was associated with CVD among the patients with DM in the present study. This finding is similar to that of the Memory in Diabetes sub-study of the Action to Control Cardiovascular Risk in Diabetes study, which reported that cognitive impairment is associated with an increased risk of CVD. ${ }^{44)}$

Functional impairment is one of the most serious geriatric symptoms. ${ }^{7)}$ A cross-sectional study of 6,588 community-dwelling individuals aged $\geq 60$ years demonstrated a 2 - to 3 -fold higher risk of functional disability in patients with $\mathrm{DM}^{45)}$ In the Women's Health and Aging Study, DM was associated with a 1.6-fold increased risk of functional disability in domains such as bathing, transfer from bed to chair, using the toilet, dressing, and eating. ${ }^{46)}$ The Study of Osteoporosis Fractures reported that women aged $\geq 65$ years with DM had a 2- to 2.5 -fold increased incidence of functional disability. ${ }^{47)}$ The results of the present study are similar to those of previous studies. In this study, functional impairment was associated with an increased prevalence of CVD and CKD. However, since CVD and CKD are important contributors to functional disabilities in patients with $\mathrm{DM}$, a prospective study is necessary to assess the causal relationship.

Notably, the present study showed that the number of geriatric syndromes was associated with CVD and CKD in patients with $\mathrm{DM}$, a finding that indicates the cumulative effects of multiple geriatric syndromes and that forms the basis of the current guideline that recommends screening for geriatric syndrome in older adults with $\mathrm{DM}^{5,8)}$

To our knowledge, the present study is the first to use a large representative sample to assess geriatric syndromes in Korean patients with DM. However, this study has several limitations. First, because of the cross-sectional nature, further prospective studies are needed to assess the causal relationship between geriatric syndromes and diabetic complications. Second, considering the lack of laboratory data and diagnoses made on the basis of responses to structured questionnaires, the prevalence of underlying diseases might be underestimated.

In conclusion, the results of this study demonstrate a higher prevalence of geriatric syndromes among older adults with DM and also confirm the association of CVD and CKD and multiple geriatric syndromes in these patients. This result is meaningful, as it forms the basis of the current DM guidelines that recommend the assessment of geriatric syndromes in older patients with DM. However, further prospective studies are needed to assess the correlation between geriatric syndromes and prognosis in patients with DM.

\section{CONFLICT OF INTEREST DISCLOSURES}

The researchers claim no conflicts of interest. 


\section{REFERENCES}

1. He W, Goodkind D, Kowal P. An aging world: 2015. International population reports. Washington: United States Census Bureau; 2016.

2. Korea Statistical Information System. Population structure by age and sex [Internet]. Daejeon: Statistics Korea; c2019 [cited 2019 Oct 31]. Available from: http://www.index.go.kr/potal/ main/EachDtlPageDetail.do?idx_cd = 1010 .

3. Jang IY, Lee HY, Lee E; The 50th Anniversary Committee of Korean Geriatrics Society. Geriatrics Fact Sheet in Korea 2018 from National Statistics. Ann Geriatr Med Res 2019;23:50-53.

4. Cho NH, Shaw JE, Karuranga S, Huang Y, da Rocha Fernandes JD, Ohlrogge AW, et al. IDF Diabetes Atlas: Global estimates of diabetes prevalence for 2017 and projections for 2045. Diabetes Res Clin Pract 2018;138:271-81.

5. Korean Diabetes Association. Treatment guideline of diabetes. 6th ed. Seoul: Korean Diabetes Association; 2019.

6. Korean Diabetes Association. Elderly diabetes mellitus. 3rd ed. Seoul: Korean Diabetes Association; 2018.

7. Araki A, Ito H. Diabetes mellitus and geriatric syndromes. Geriatr Gerontol Int 2009;9:105-14.

8. American Diabetes Association. 12. Older adults: standards of medical care in diabetes-2019. Diabetes Care 2019;42(Suppl 1):S139-S147.

9. Kirkman MS, Briscoe VJ, Clark N, Florez H, Haas LB, Halter JB, et al. Diabetes in older adults. Diabetes Care 2012;35:2650-64.

10. Won CW, Yoo HJ, Yu SH, Kim CO, Dumlao LC, Dewiasty E, et al. Lists of geriatric syndromes in the Asian-Pacific geriatric societies. Eur Geriatr Med 2013;4:335-8.

11. Yoo HJ. Clinical implications of geriatric syndromes. J Korean Med Assoc 2014;57:738-42.

12. Bourdel-Marchasson I, Berrut G. Caring the elderly diabetic patient with respect to concepts of successful aging and frailty. Diabetes Metab 2005;31 Spec No 2:5S13-5S19.

13. Inouye SK, Studenski S, Tinetti ME, Kuchel GA. Geriatric syndromes: clinical, research, and policy implications of a core geriatric concept. J Am Geriatr Soc 2007;55:780-91.

14. Chung H. 2017 National Survey of Older Koreans: findings and implications. Sejong: Korea Institute for Health and Social Affairs; 2018.

15. Masnoon N, Shakib S, Kalisch-Ellett L, Caughey GE. What is polypharmacy? A systematic review of definitions. BMC Geriatr 2017;17:230.

16. Brown RT, Diaz-Ramirez LG, Boscardin WJ, Lee SJ, Steinman MA. Functional impairment and decline in middle age: a cohort study. Ann Intern Med 2017;167:761-8.
17. Johnson JK, Lui LY, Yaffe K. Executive function, more than global cognition, predicts functional decline and mortality in elderly women. J Gerontol A Biol Sci Med Sci 2007;62:1134-41.

18. Al-Musawe L, Martins AP, Raposo JF, Torre C. The association between polypharmacy and adverse health consequences in elderly type 2 diabetes mellitus patients; a systematic review and meta-analysis. Diabetes Res Clin Pract 2019;155:107804.

19. Shorr RI, Ray WA, Daugherty JR, Griffin MR. Incidence and risk factors for serious hypoglycemia in older persons using insulin or sulfonylureas. Arch Intern Med 1997;157:1681-6.

20. Field TS, Gurwitz JH, Harrold LR, Rothschild J, DeBellis KR, Seger AC, et al. Risk factors for adverse drug events among older adults in the ambulatory setting. J Am Geriatr Soc 2004; 52:1349-54.

21. Marcum ZA, Amuan ME, Hanlon JT, Aspinall SL, Handler SM, Ruby CM, et al. Prevalence of unplanned hospitalizations caused by adverse drug reactions in older veterans. J Am Geriatr Soc 2012;60:34-41.

22. Doan J, Zakrzewski-Jakubiak H, Roy J, Turgeon J, Tannenbaum C. Prevalence and risk of potential cytochrome P450-mediated drug-drug interactions in older hospitalized patients with polypharmacy. Ann Pharmacother 2013;47:324-32.

23. Johnell K, Klarin I. The relationship between number of drugs and potential drug-drug interactions in the elderly: a study of over 600,000 elderly patients from the Swedish Prescribed Drug Register. Drug Saf 2007;30:911-8.

24. Fitzgerald SP, Bean NG. An analysis of the interactions between individual comorbidities and their treatments: implications for guidelines and polypharmacy. J Am Med Dir Assoc 2010; 11:475-84.

25. Noale M, Veronese N, Cavallo Perin P, Pilotto A, Tiengo A, Crepaldi G, et al. Polypharmacy in elderly patients with type 2 diabetes receiving oral antidiabetic treatment. Acta Diabetol 2016;53:323-30.

26. Lipska KJ, Krumholz H, Soones T, Lee SJ. Polypharmacy in the aging patient: a review of glycemic control in older adults with type 2 diabetes. JAMA 2016;315:1034-45.

27. Schwartz AV, Hillier TA, Sellmeyer DE, Resnick HE, Gregg E, Ensrud KE, et al. Older women with diabetes have a higher risk of falls: a prospective study. Diabetes Care 2002;25:1749-54.

28. Volpato S, Leveille SG, Blaum C, Fried LP, Guralnik JM. Risk factors for falls in older disabled women with diabetes: the women's health and aging study. J Gerontol A Biol Sci Med Sci 2005; 60:1539-45.

29. Petrofsky JS, Cuneo M, Lee S, Johnson E, Lohman E. Correlation between gait and balance in people with and without Type 2 diabetes in normal and subdued light. Med Sci Monit 2006; 


\section{2:CR273-81.}

30. Arvanitakis Z, Wilson RS, Schneider JA, Bienias JL, Evans DA, Bennett DA. Diabetes mellitus and progression of rigidity and gait disturbance in older persons. Neurology 2004;63:996-1001.

31. Menz HB, Lord SR, St George R, Fitzpatrick RC. Walking stability and sensorimotor function in older people with diabetic peripheral neuropathy. Arch Phys Med Rehabil 2004;85:245-52.

32. Brown JS, Vittinghoff E, Lin F, Nyberg LM, Kusek JW, Kanaya AM. Prevalence and risk factors for urinary incontinence in women with type 2 diabetes and impaired fasting glucose: findings from the National Health and Nutrition Examination Survey (NHANES) 2001-2002. Diabetes Care 2006;29:1307-12.

33. Lifford KL, Curhan GC, Hu FB, Barbieri RL, Grodstein F. Type 2 diabetes mellitus and risk of developing urinary incontinence. $J$ Am Geriatr Soc 2005;53:1851-7.

34. Jackson SL, Scholes D, Boyko EJ, Abraham L, Fihn SD. Urinary incontinence and diabetes in postmenopausal women. Diabetes Care 2005;28:1730-8.

35. Kwon CS, Lee JH. Prevalence, risk factors, quality of life, and health-care seeking behaviors of female urinary incontinence: results from the 4th Korean National Health and Nutrition Examination Survey VI (2007-2009). Int Neurourol J 2014;18:316.

36. Park J, Hong GR, Yang W. Factors associated with self-reported and medically diagnosed urinary incontinence among community-dwelling older women in Korea. Int Neurourol J 2015; 19:99-106.

37. Perlmuter LC, Hakami MK, Hodgson-Harrington C, Ginsberg J, Katz J, Singer DE, et al. Decreased cognitive function in aging non-insulin-dependent diabetic patients. Am J Med 1984; 77:1043-8.

38. Reaven GM, Thompson LW, Nahum D, Haskins E. Relationship between hyperglycemia and cognitive function in older NIDDM patients. Diabetes Care 1990;13:16-21.

39. Ott A, Stolk RP, Hofman A, van Harskamp F, Grobbee DE, Breteler MM. Association of diabetes mellitus and dementia: the Rotterdam Study. Diabetologia 1996;39:1392-7.

40. Cukierman T, Gerstein HC, Williamson JD. Cognitive decline and dementia in diabetes--systematic overview of prospective observational studies. Diabetologia 2005;48:2460-9.

41. Araki A, Ito H. Glucose metabolism, advanced glycation endproducts, and cognition. Geriatr Gerontol Int 2004;4:S108 -S110.

42. Langan SJ, Deary IJ, Hepburn DA, Frier BM. Cumulative cognitive impairment following recurrent severe hypoglycaemia in adult patients with insulin-treated diabetes mellitus. Diabetologia 1991;34:337-44.

43. Kalmijn S, Feskens EJ, Launer LJ, Stijnen T, Kromhout D. Glucose intolerance, hyperinsulinaemia and cognitive function in a general population of elderly men. Diabetologia 1995;38:1096102.

44. Stefanidis KB, Askew CD, Greaves K, Summers MJ. The effect of non-stroke cardiovascular disease states on risk for cognitive decline and dementia: a systematic and meta-analytic review. Neuropsychol Rev 2018;28:1-15.

45. Gregg EW, Beckles GL, Williamson DF, Leveille SG, Langlois JA, Engelgau MM, et al. Diabetes and physical disability among older U.S. adults. Diabetes Care 2000;23:1272-7.

46. Volpato S, Blaum C, Resnick H, Ferrucci L, Fried LP, Guralnik JM, et al. Comorbidities and impairments explaining the association between diabetes and lower extremity disability: the Women's Health and Aging Study. Diabetes Care 2002;25:678-83.

47. Gregg EW, Mangione CM, Cauley JA, Thompson TJ, Schwartz AV, Ensrud KE, et al. Diabetes and incidence of functional disability in older women. Diabetes Care 2002;25:61-7. 